\title{
Avaliação de modelos não-lineares e da relação do consumo voluntário de vacas primíparas e de bezerros com a curva de lactação de vacas Nelore ${ }^{1}$
}

\section{Lara Toledo Henriques ${ }^{2}$, Sebastião de Campos Valadares Filho ${ }^{3}$, Mozart Alves Fonseca ${ }^{2}$, Pedro Veiga Rodrigues Paulino ${ }^{3}$, Edenio Detmann ${ }^{3}$, Rilene Ferreira Diniz Valadares ${ }^{4}$}

\author{
1 Projeto financiado pelo CNPq , FAPEMIG e CAPES \\ 2 Programa de Pós-graduação em Zootecnia-UFV. \\ ${ }^{3}$ Departamento de Zootecnia - UFV. \\ ${ }^{4}$ Departamento de Medicina Veterinária - UFV
}

RESUMO - Procurou-se avaliar a precisão de cinco modelos não-lineares em descrever a forma da curva de produção de leite de vacas Nelore e o efeito do consumo voluntário (CV) da vaca e do bezerro sobre a produção de leite (PL). Foram testados os modelos de Sikka, Nelder, Wood, Jenkins \& Ferrell, e Jenkins \& Ferrell com um parâmetro de ajustamento. Foram utilizadas 12 vacas primíparas com peso corporal médio de $359 \mathrm{~kg}( \pm 8)$ e seus respectivos bezerros. A produção de leite foi estimada pela pesagem do bezerro antes e após a mamada, do nascimento aos 180 dias de idade. As pesagens foram efetuadas duas vezes ao dia, semanalmente, após 6 horas de jejum de líquido e sólidos. Os modelos não-lineares de Sikka, Jenkins \& Ferrell, Nelder e Wood não descreveram a curva de lactação apropriada devido ao excesso ou subestimação d o pico da produção de leite. O melhor ajustamento foi encontrado para o modelo de Jenkins \& Ferrell com um parâmetro de ajustamento. O efeito do consumo voluntário da vaca e do bezerro, avaliado separadamente, não se correlacionou com a produção de leite. Entretanto, ao avaliar o consumo da vaca e do bezerro conjuntamente, foi encontrada uma correlação positiva e negativa com a produção de leite, respectivamente. A produção de leite está intimamente correlacionada com o consumo da vaca e do bezerro, e a capacidade de ingerir sólidos não lácteos reulta na redução da necessidade de leite da mãe.

Palavras-chave: persistência, produção de leite

\section{Evaluation of non-linear models and the effects of primiparous cows and calves intake on the lactation curve of Nelore cows}

\begin{abstract}
This research was carried out to evaluate five non-linear mathematical models to describe lactation curves of Nelore cows and effect of the cow and calf intake on milk yield. In this study we compared the models of Sikka (1950), Nelder (1966), Wood (1967), Jenkins \& Ferrell (1984) and Jenkins \& Ferrell (1984) with a fit parameter. Data of production were collected from 12 primiparous cows with a mean live weight of $359 \mathrm{~kg}( \pm 8)$ and its offspring. The milk production was estimated weighing the calf before and after suckling, from birth to 180 days of age. Measurements were made twice a day in a weekly period after 6 hour fasting of liquid and solids. The mathematical models of Sikka, Jenkins \& Ferrell, Nelder and Wood failed to describe lactation curve appropriated due to over or underestimation of milk yield peak. Better adjustement was found for the model of Jenkins \& Ferrell with a fitting parameter. Cow and calf intake evaluated separately was no correlated with milk yield. Conversely at evaluating both cow and calf together, it was found a positive and a negative correlation with milk yield, respectively. Milk yield is is closely correlated wiht cow and calf intake, and the capacity of the calf to ingest non-dairy solids results in reduction of milk needs from dam.
\end{abstract}

Key Words: milk production, persistency

\section{Introdução}

O potencial de produção de leite de animais do rebanho de corte no Brasil passou na última década a ser foco de grande atenção à medida que os sistemas de criação se intensificaram. A abertura do mercado mundial para exportação decorreu na criação de diretrizes em qualidade de carne, onde a redução da idade de abate passou a ser exigência de mercado.

O peso ao desmame, que constitui reflexo do volume de produção de leite da vaca, bem como da qualidade deste leite, é um dos indicadores de produção. Estudos têm demonstrado que a correlação fenotípica entre a produção de leite da vaca e o peso à desmama do bezerro é alta e 
positiva, indicando que vacas de maior produção de leite desmamam bezerros mais pesados. Ainda neste sentido, em sistemas de criação mais intensivos de produção, bezerros mais pesados à desmama são desejados, pois representam redução na idade de abate dos machos e na idade à puberdade das fêmeas.

Atrelado a isto, nota-se disponibilidade reduzida de informações sobre a produção de leite de animais zebuínos, principalmente em condições tropicais. Sabe-se, contudo, que a curva de lactação de animais de corte apresenta o pico mais tardiamente que animais oriundos de rebanhos leiteiros, ocorrendo ao redor da sexta semana pós-parto (Jenkins \& Ferrell, 2004).

A descrição da curva de lactação, bem como a estimação de volume de leite que estará a disposição dos bezerros, são fortes ferramentas para se definir melhor a estratégia de suplementação do rebanho. Desta forma, como a estimativa de ganho de peso está associada à expectativa da idade de abate, pode-se efetuar o planejamento nutricional para produção de carne, da lactação à terminação.

A primeira referência de um modelo de curva de lactação foi atribuída a Brody et al. (1923). Porém, foi Wood (1967) quem propôs a introdução da função gama em modelos determinísticos, para melhor ajustamento das fases ascendentes e descendentes da curva com o auxílio de conceitos probabilísticos. A partir desse estudo, outros modelos foram propostos. No entanto, a habilidade de tais modelos matemáticos para descrever a curva de lactação de animais zebuínos em condições tropicais carece de investigação.

Desta forma, avaliou-se o ajuste de cinco modelos matemáticos não lineares em descrever a forma da curva de lactação de vacas primíparas Nelore, bem como a relação entre o consumo voluntário da vaca e do bezerro com a produção de leite.

\section{Material e Métodos}

O experimento foi realizado no Departamento de Zootecnia e no Laboratório de Nutrição Animal pertencentes à Universidade Federal de Viçosa, cidade de Viçosa, estado de Minas Gerais, Brasil. Foram utilizados 12 vacas primíparas Nelore, com peso corporal médio inicial de $359 \pm 8$ kg, e seus respectivos bezerros. A pesagem das vacas foi realizada a cada 28 dias.

A área experimental para cada par vaca/bezerro consistia de 33,84 $\mathrm{m}^{2}$ com bebedouro e comedouro, sendo este vedado ao acesso dos bezerros, e uma outra área de igual dimensão de acesso exclusivo dos bezerros em sistema creep feeding.
A dieta fornecida às vacas primíparas foi formulada de acordo com recomendações do NRC (1996), utilizando-se silagem de milho como volumoso e concentrado formulado à base de fubá de milho, farelo de soja e uréia, na proporção 70:30 volumoso:concentrado (base da matéria seca), respectivamente (Tabela 1). A dieta foi ofertada ad libtum (permitindo-se sobras de $10 \%$ da quantidade ofertada) em dois tratos diários. A dieta dos bezerros foi composta pelo leite e, após o $1^{1}$ mês de lactação, foi ofertada silagem de milho e concentrado comercial ad libitum.

A produção de leite foi obtida por intermédio da pesagem do bezerro em balança digital com precisão de 100 g, antes e após a mamada conforme McCarter et al. (1991), até estes completarem 180 dias. Os procedimentos de pesagens dos bezerros se iniciavam às 18 horas do dia anterior, quando estes eram apartados de suas mães e mantidos em jejum até as 6 horas da manhã seguinte, quando eram novamente colocados junto às suas mães para mamar e esvaziar completamente o úbere. Após este período, os bezerros permaneciam por 6 horas em jejum de líquidos e sólidos e finalmente às 12 horas eram pesados, colocados para mamar, e novamente pesados, estimando-se assim a produção de leite por 6 horas. Em seguida, os bezerros eram mantidos novamente em jejum até as 18 horas quando se procedia à pesagem antes e após a mamada, com mais uma estimativa de produção de leite por 6 horas. As pesagens foram realizadas uma vez por semana, entre a $1^{\mathrm{a}}$ e a $26^{\mathrm{a}}$ semana de vida.

A estimativa da produção de leite por dia $\left(P L D_{n}\right.$ em 24 horas) foi feita pelo somatório das diferenças de peso dos bezerros nas duas pesagens, multiplicada por dois: $P L D s=(P 1+P 2) \times 2$, em que: $s$ representa a semana de lactação (variando de 1 a 26) e P1 e P2 referem-se a diferença de peso obtido na primeira e segunda pesagens dos bezerros.

A produção de leite total ( $P L T)$, estimada do nascimento aos 180 dias de idade foi obtida pela soma das observações dos dias de pesagem multiplicada por sete (sete dias na semana): $P L T=\sum_{n=1}^{26}(P L D n \times 7)$ em que: $P L D n$ segue a definição anterior.

A persistência da lactação (PER) foi estimada pela relação da diferença entre a última produção de leite diária e a mais alta produção verificada:

$P E R=(P L D f-P L D$ pico $) / n$ em que: $P L D f$ é produção de leite obtida no final da lactação, $P L D$ pico é a mais alta produção de leite verificada e $n$ é o número de dias observado entre o pico e o final da lactação. Esta variável é expressa em g/d ${ }^{-1}$ e representa o quanto se reduz a produção média de leite por dia, a partir do pico de lactação (Jenkins et al., 2000). 
Foram coletadas diariamente amostras de alimentos fornecidos e de sobras, as quais foram posteriormente agrupadas por animal em compostas semanais para avaliação do consumo de matéria seca das vacas e dos bezerros. Todas as amostras foram submetidas à secagem em ventilação forçada ( $60^{\circ} / 72$ horas) e moídas em peneira com crivos de $1 \mathrm{~mm}$. Foram quantificados os teores de matéria seca (MS), matéria mineral (MM), proteína bruta (PB), extrato etéreo (EE), fibra em detergente ácido (FDA) e a lignina $\left(\mathrm{H}_{2} \mathrm{SO}_{4} 72 \%\right.$ p/p) de acordo com recomendações de Silva \& Queiroz (2002). As concentrações de fibra em detergente neutro (FDN) e nitrogênio insolúvel em detergente neutro (NIDN) foram determinadas de acordo com os protocolos descritos por Mertens (2002) e Licitra et al. (1996). A estimativa do consumo voluntário das vacas foi realizada por intermédio dos teores de fibra em detergente neutro indigestível (FDNi), os alimentos permaneceram em sacos confeccionados em tecido não-tecido (TNT-100 g/m²) por 240 h no rúmen de um bovino mestiço, alimentado com dieta similar a oferecida às vacas primíparas. Após este período, os sacos foram retirados do rúmen, lavados até total clareamento e submetidos a extração com detergente neutro (Mertens, 2002). Os carboidratos não-fibrosos do concentrado foram calculados segundo Hall (2000) em que, $\% \mathrm{CNF}=100-[(\% \mathrm{~PB}-\% \mathrm{~PB}$ derivada da uréia+\% de uréia $)$ $+\% \mathrm{EE}+\% \mathrm{FDN}+\%$ cinzas].

Os modelos matemáticos testados para descrever a curva de lactação dos animais seguiram os propostos por:

Sikka (1950), com o modelo exponencial;

$\mathrm{Y} t=a \cdot \exp \left(b s-c s^{2}\right)$

em que: $\mathrm{Y} t=$ produção de leite; $a=$ fator de escala que expressa a fase inicial da lactação; exp = função exponencial; $b=$ fator associado à inclinação da curva que expressa a fase ao redor do pico da lactação; $c$ = parâmetro de estimação que define a persistência da lactação; e $s$ = semana de lactação.

Nelder (1966), com o modelo polinomial inverso; $\mathrm{Y} t=s . /\left(a+b s+c s^{2}\right)$

em que: $\mathrm{Y} t, a, b$, e $c$ seguem as definições anteriores.
Wood (1967), com o modelo que introduziu a função gama;

$\mathrm{Y} t=a s^{b} \cdot \exp ^{-c s}$

em que: Yt, $a, s, b$, exp e $c$ seguem as definições anteriores. Jenkins \& Ferrell (1984) com a introdução da função gama e com o expoente $b$ suprimido;

$\mathrm{Y} t=a s . \exp ^{-c s}$

em que: Yt, $a$, $s$, exp e $c$ seguem as definições anteriores. Jenkins \& Ferrell (1984) propondo-se modificação com a adição de um parâmetro de ajustamento para o início da lactação;

$\mathrm{Y} t=a+b s . \exp ^{-c s}$

em que: Yt, $a, b$, s, exp e $c$ seguem as definições anteriores.

A qualidade do ajustamento das equações foi avaliada por intermédio do desvio-padrão assintótico e pela dispersão de resíduos padronizados (Souza, 1998).

Os valores de desvio-padrão assintótico foram estimados pelo método da máxima verossimilhança:

$D P A=\sqrt{\frac{\sum_{i}^{1}\left(y_{i}-\hat{y}_{i}\right)}{n}}$

em que: $D P A=$ desvio-padrão assintótico; $y_{i}=$ valor observado $\mathrm{i} ; \hat{y}_{i}=$ valor estimado $\mathrm{i}$; e $n=$ número de observações.

Os resíduos padronizados foram obtidos a partir da relação: $R P_{i}=\frac{y_{i}-\hat{y}_{i}}{D P A}$

em que $R P_{i}$ é o resíduo padronizado i.

Os efeitos do consumo voluntário (CV) das vacas primíparas, do consumo voluntário de sólidos dos bezerros e os pesos dos animais sobre a produção de leite, foram avaliados por análise de regressão linear e não-linear (Souza, 1998).

De forma particular o CV advindo de concentrado e volumoso dos bezerros foi ajustado para semana de início de ingestão de sólidos:

$\hat{Y}=\exp [k(s-a)]$

Tabela 1- Composição da silagem de milho, dos concentrados e da silagem das vacas primíparas e dos bezerros com base na matéria seca

\begin{tabular}{lccc}
\hline Itens & Silagem de milho & Concentrado vacas & Concentrado bezerros \\
\hline Matéria seca (\%) & 33,06 & 84,67 & 88,39 \\
Matéria mineral (\%MS) & 8,0 & 4,39 & 9,85 \\
Proteína bruta (\%MS) & 6,31 & 19,24 & 22,51 \\
Fibra em detergente neutro (\%MS) & 60,93 & 12,06 & 30,73 \\
Fibra em detergente neutro corrigida para proteína (\%MS) & 57,76 & 11,22 & 16,71 \\
Carboidratos não-fibrosos (\%MS) & 24,38 & 72,29 & - \\
Fibra em detergente neutro indigestível (\%MS) & 22,26 & 1,66 & 5,34 \\
Fibra em detergente ácido (\%MS) & 38,70 & 1,85 & 4,36 \\
Lignina (\%MS) & 5,51 & 3,91 & 3,51 \\
Extrato etéreo (\%MS) & 3,56 & & 3,57 \\
\hline
\end{tabular}


em que: $k=$ taxa de incremento de consumo na semana $\left(s^{-1}\right)$; e $a$ = parâmetro de ajustamento, sem interpretação biológica direta.

A avaliação da influência dos consumos voluntários da vaca e do bezerro sobre a produção de leite foi realizada por análise de trilha, segundo Cruz \& Regazzi (2001), com auxílio do programa SAEG - Sistema para Análises Estatísticas, versão 9.1 (UFV 2007). Todas as análises foram realizadas utilizando-se o Statistical Analysis System (SAS Institute, 1989).

\section{Resultados e Discussão}

A representação gráfica da produção de leite é a curva de lactação que consiste, sumariamente, de parâmetros de produção de leite determinados pela eficiência biológica da vaca e pode ser usada como parâmetro para seleção e manejo nutricional (Sherchand et al., 1995). Os modelos estimados por valores anteriormente mensurados de produção de leite podem ser usados para predizer a futura produção de leite para o bezerro.

Os modelos não-lineares de Sikka (1950) e Jenkins \& Ferrell (1984) não descreveram a produção de leite das vacas Nelore, devido a falta de ajustamento dos mesmos (Figura 1). Segundo Sherchand et al. (1995), o modelo de Sikka (1950) se ajusta bem aos dados no início da lactação, mas o mesmo não ocorre no final da lactação, possivelmente devido à função exponencial ajustar-se bem ao redor do pico. O modelo de Sikka (1950) apresentou o pico de lactação mais tardio ( $11^{\text {a }}$ sem) dentre os modelos avaliados. O modelo de Jenkins \& Ferrell (1984) inclinou a curva de forma demasiada até o pico de produção, sendo esta exacerbação descrita por Landete-Castillejos \& Gallego (2000) como decorrência da polinomial inversa.

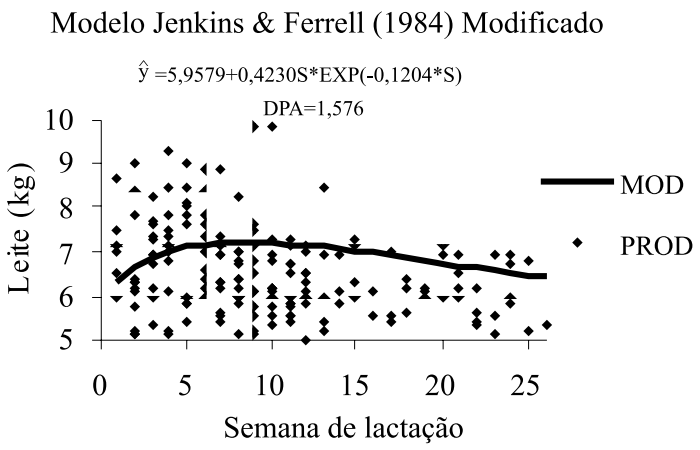

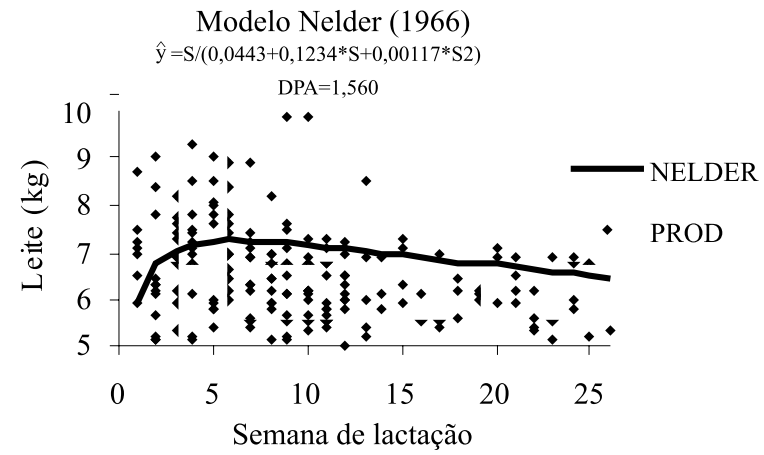

Modelo Jenkins \& Ferrell (1984)

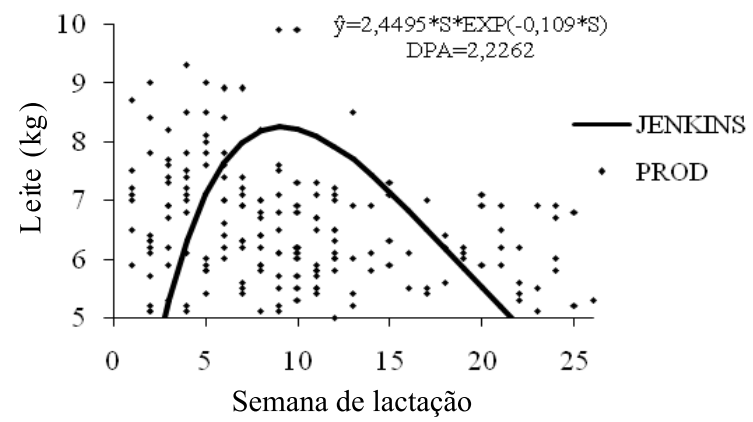

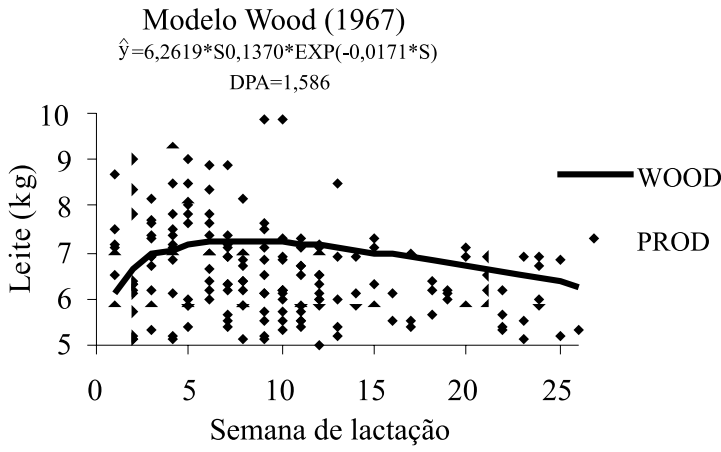

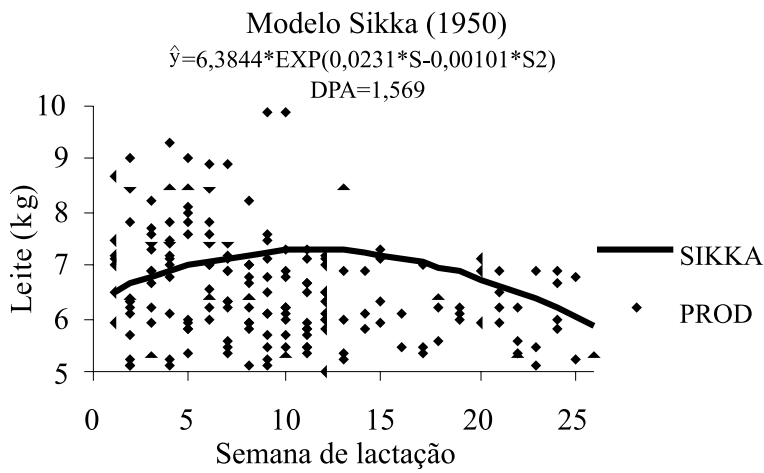

Figura 1 - Descrição das curvas de lactação segundo os modelos testados. 
O modelo de Nelder (1966) estimou o pico da lactação extemporâneo, ou seja, em outro momento da curva, quando comparado com os demais modelos testados, o que pode ter sido devido à função polinomial inversa, uma vez que este modelo se ajusta bem em lactações superiores a 44 semanas (Sherchand et al., 1995).

O parâmetro $c$ da curva de lactação, segundo Jenkins \& Ferrell (1984, geralmente não afeta a forma da curva, mas pode ser uma medida indireta da persistência da lactação. Assim, o modelo de Nelder (1966) estimou, dentre os modelos testados, a maior persistência de lactação. A persistência é definida como a diferença da produção no último dia de lactação e a produção no pico da lactação, relativo ao número de dias entre os dois eventos (Jenkins et al., 2000).
No modelo de Wood (1967), a função $s^{b}$ permite integrar a fase ascendente da lactação e o termo exponencial torna completa a fase decrescente da curva (Masselin et al., 1987). Os parâmetros b e c do modelo de Wood (1967) são indicados como índices da fase ascendente e descendente da curva de produção de leite, respectivamente. Assim, conforme a equação ajustada (Figura 1) observaram-se baixos índices tanto para o parâmetro $b(0,1370)$ quanto para o parâmetro $c(0,0171)$, o que resultou em um pico de lactação menos proeminente, resultando em falta de ajuste do modelo.

A avaliação dos modelos baseou-se nos desvios-padrão assintóticos e nas dispersões dos resíduos padronizados (Figura 2). Os modelos de Sikka (1950), Jenkins \& Ferrell (1984), Nelder(1996), Wood(1967) e Jenkins \& Ferrell (1984)

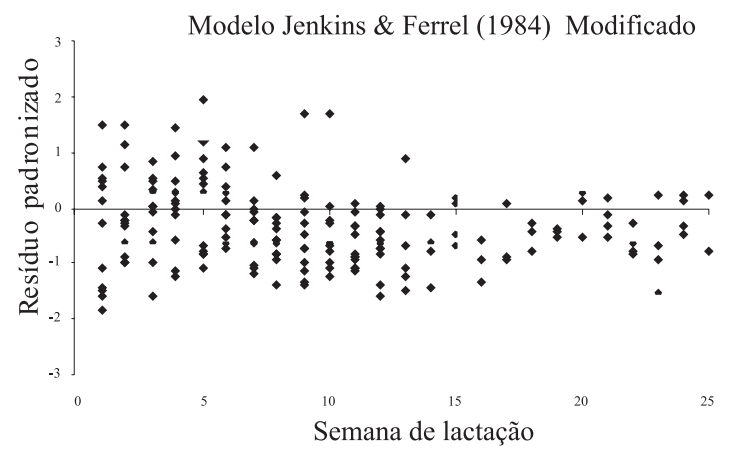

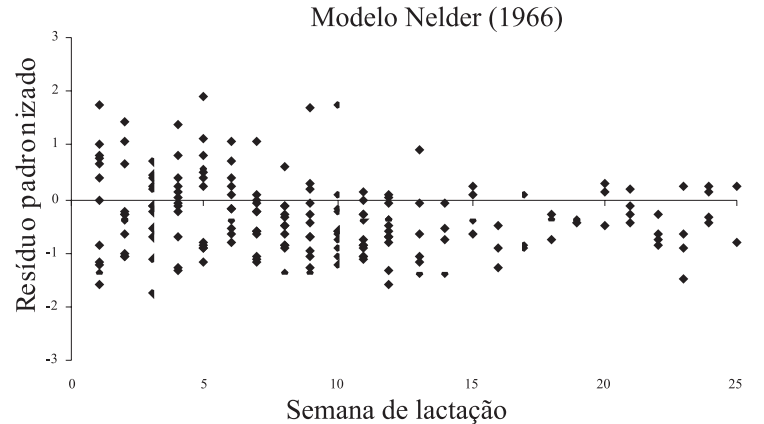

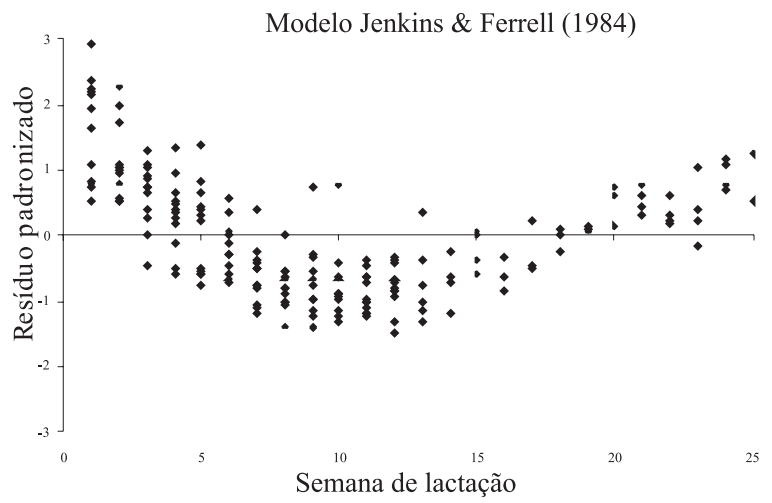

Figura 2 - Resíduos padronizados dos modelos avaliados.
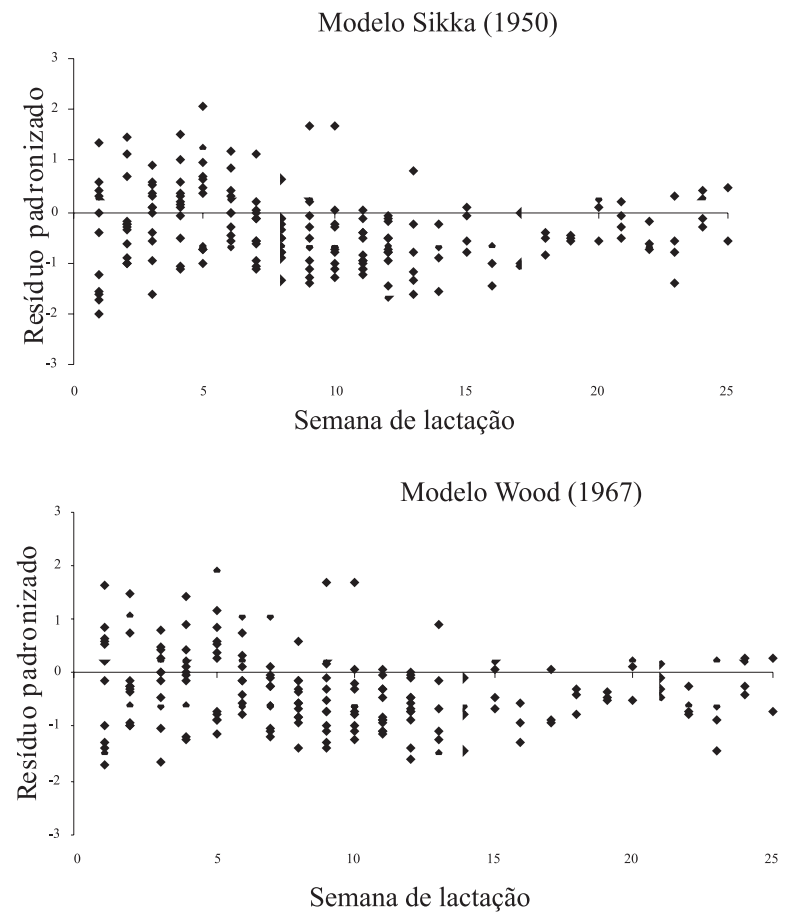
Modificado apresentaram os seguintes DPA: 1,569; 2,226; 1,560; 1,586 e 1,576; respectivamente.

Baixos desvios-padrão assintóticos são indicativos de boa qualidade de ajustamento do modelo ao conjunto de dados. Partindo então desta premissa, descartou-se o modelo de Jenkins \& Ferrell (1984) por apresentar DPA muito elevado em comparação aos demais.

A inspeção do padrão de comportamento dos resíduos padronizados (Figura 2) auxilia no diagnóstico de ajustamento dos modelos ao conjunto de dados. O padrão de bom ajustamento é aquele que apresenta o conjunto de resíduos com dispersão regular em torno dos eixos das abscissas, o qual representa a variável independente (Draper \& Smith, 1966), ou seja, semana de lactação.

Os modelos de Wood (1967) e Jenkins \& Ferrell (1984) Modificado foram os que apresentaram maior número de pontos dispersos na faixa de 1 a -1 (77,91\%) dos resíduos padronizados (Tabela 2). Assume-se, assim, que, com base na dispersão dos resíduos padronizados, esses modelos foram os que mais se adequaram aos dados de produção de leite estudados. No entanto, na análise da conformação da curva e da projeção do início da lactação e do pico de lactação, o modelo de Jenkins \& Ferrell (1984) Modificado apresentou a melhor conformação, por apresentar uma distribuição mais regular em torno do eixo das abscissas. Desta forma, assume-se que o modelo que mais se adequou ao conjunto de dados foi o de Jenkins \& Ferrell (1984) Modificado.

Após avaliação dos modelos, estabeleceu-se que a curva de produção de leite gerada pelo modelo de Jenkins \& Ferrell (1984) com o parâmetro de ajustamento, seria a curva utilizada para subseqüente determinação dos dados de produção de leite.

O pico da lactação das vacas primíparas ocorreu na 8 a semana pós-parto com estimativa média observada de 7,25 kg/dia. Segundo Jenkins \& Ferrell (1984), o pico de lactação de animais de raças especializadas em produção de carne é mais tardio que em animais de origem leiteira. Isto poderia ser atribuído à presença constante do bezerro ao lado da mãe, que, ao se tornar mais pesado, apresenta maior capacidade de ingestão e passa a exigir mais da vaca.

Tabela 2 - Distribuição acumulada dos resíduos padronizados

\begin{tabular}{lccccc}
\hline \multicolumn{5}{c}{ Modelos } \\
\hline Item (\%) & Sikka & J \& F & Nelder & Wood & J \& F Mod \\
\hline 3 a 1 & 6,40 & 13,37 & 6,40 & 5,81 & 5,81 \\
1 a 0 & 24,41 & 34,88 & 25,58 & 25,58 & 26,75 \\
0 a -1 & 50,00 & 38,88 & 50,58 & 52,33 & 51,16 \\
-1 a -3 & 19,19 & 13,37 & 17,44 & 16,28 & 16,28 \\
\hline
\end{tabular}

J\&F = Jenkins \& Ferrell; J\&F Mod = Jenkins \& Ferrell Modificado.
Posteriormente, com o desenvolvimento ruminal e adequação microbiana à fermentação de forragem, ocorre a troca gradativa do alimento líquido pelo pasto, dependendo assim menos do leite para atender às suas exigências nutricionais.

Por outro lado, em bezerros oriundos de rebanho leiteiro, como o bezerro é separado da mãe ao nascimento, o efeito hormonal (e.g. prolactina) é menos determinante na produção de leite. No entanto, em gado de corte, o aspecto hormonal é mais relevante devido à presença constante do bezerro (Alencar et al., 1985).

A estimativa média de produção total de leite até os 180 dias (PLT) de lactação foi de 1.253,140 kg, com produção média diária (PLD) de 6,885 kg, e persistência da lactação (PER) de $-0,0064$ g.d $\mathrm{d}^{-1}$. Ressalta-se, contudo, que os animais avaliados estavam na primeira lactação e eram não gestantes.

Várias são as variáveis que influenciam a lactação de uma vaca de corte. Pimentel et al. (2005) avaliaram a lactação de vacas Hereford e Nelore gestantes ou não-gestantes e relataram que a produção de leite das gestantes foi superior à das não-gestantes, no entanto, possivelmente outras variáveis influenciaram a produção de leite. A ordem da lactação pode ser citada como uma destas variáveis, já que vacas multíparas, quando no mesmo sistema de criação, tendem a produzir mais leite.

A curva de consumo voluntário (CV) do bezerro acrescentou-se um parâmetro de ajustamento, sem interpretação biológica direta em referência às 5 semanas iniciais de vida dos bezerros, onde estes não ingeriram matéria seca advinda de volumoso ou concentrado por estarem apenas lactantes.

A substituição do leite pela forragem nos bezerros foi gradativa e ocorreu entre a $8^{\mathrm{a}}$ e a $12^{\mathrm{a}}$ semana de vida, momento em que a ingestão de MS de sólidos (concentrado e volumoso) passou a ser mais acentuada (Figura 3). O incremento semanal no consumo voluntário dos bezerros advindo dos alimentos sólidos foi baixo até 90 dias (aproximadamente 12,5 - semana), apenas 52,9 g/dia. Por outro lado, o aumento no consumo que ocorreu entre os 90 e 180 dias de vida foi de seis vezes, ou cerca de 317,4 g ao final do período (Tabela 3 e Figura 4).

O incremento semanal no consumo voluntário foi estimado pela sua derivada:

$\frac{d \hat{y}}{d x}=0,1329 \exp ^{[0,1329(x-19,45)]}$

Dessa forma, os resultados comprovaram grande dependência dos bezerros ao leite materno na fase inicial da lactação, que ocorreu entre o nascimento e a 12,5aㅗ semana de 

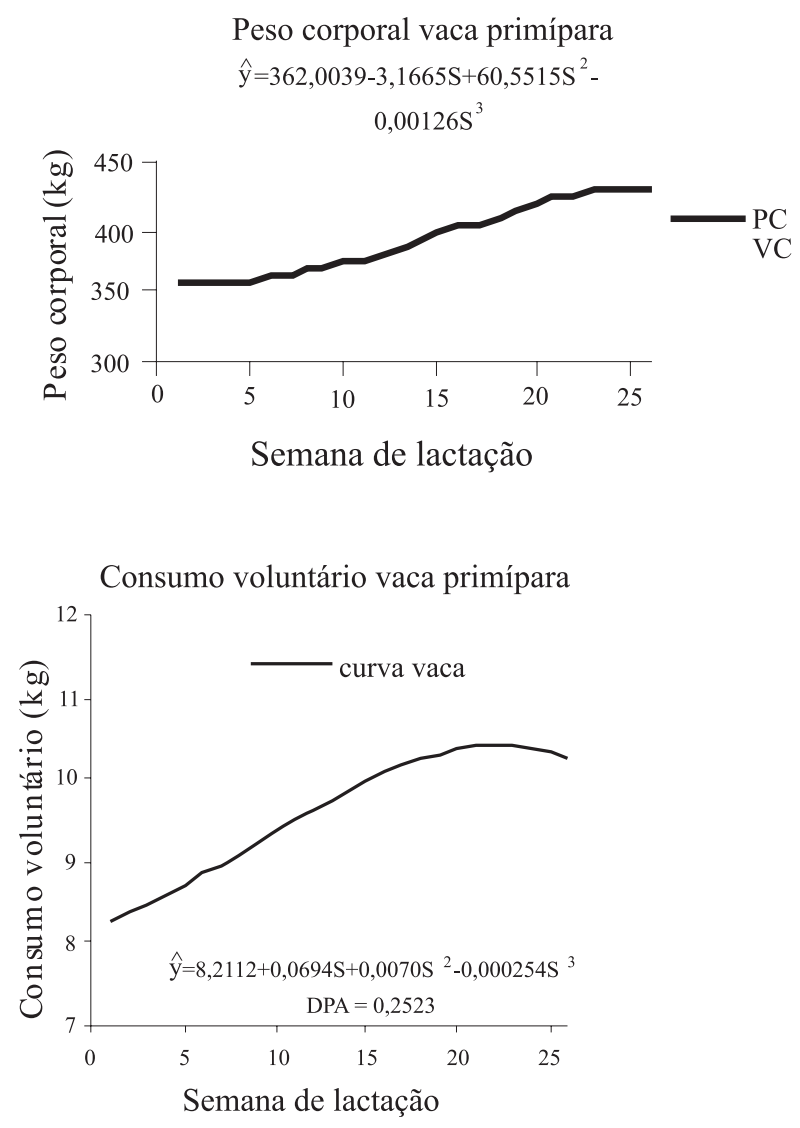

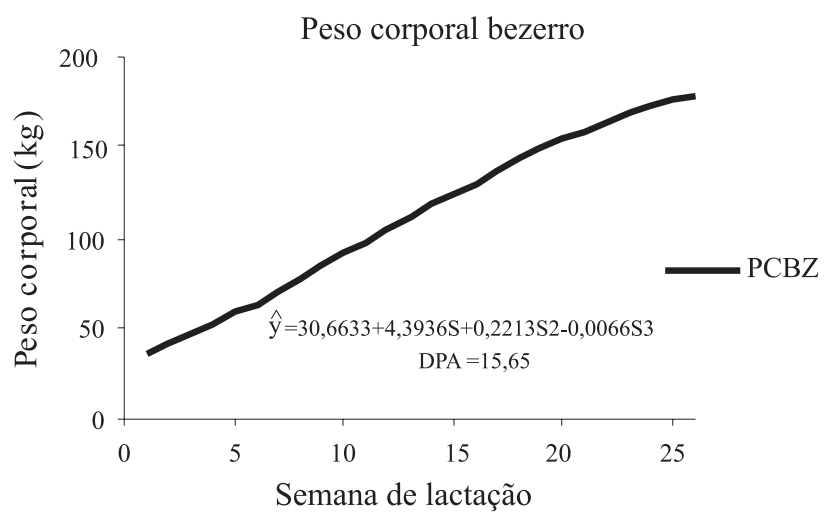

Consumo voluntário de sólidos

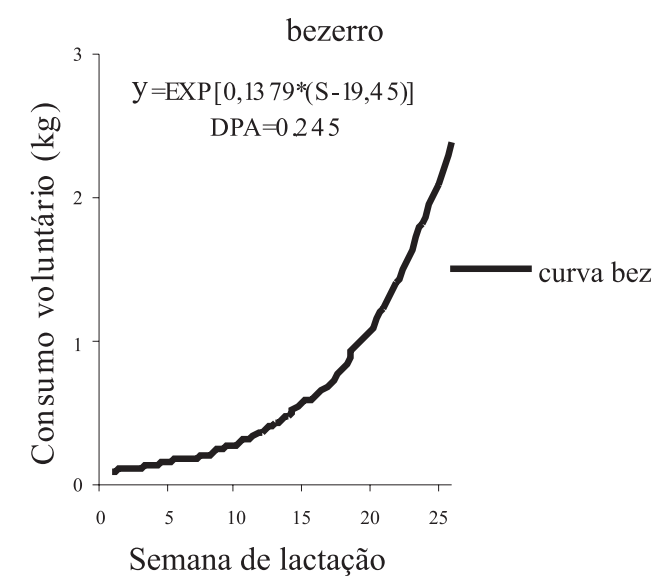

Figura 3 - Curvas do peso corporal da vaca e do bezerro e seus respectivos consumos voluntários.

Tabela 3 - Incremento do consumo voluntário (g/sem) e peso corporal médio dos bezerros (PC-kg)

\begin{tabular}{ccc}
\hline Semana de idade & Incremento no consumo voluntário & Peso corporal \\
\hline 1 & 11,4 & 33,31 \\
5 & 19,5 & 58,05 \\
10 & 37,9 & 88,78 \\
15 & 73,6 & 126,45 \\
20 & 143,0 & 149,5 \\
25 & 277,9 & 197,37 \\
& Incremento total & Ganho peso corporal médio \\
\end{tabular}

vida. No entanto, após esta fase, a dependência pelo leite se reduziu de forma mais intensa; momento no qual o peso dos animais já havia triplicado (29,5 kg vs 107,5 kg), confirmando sua maior capacidade de ingestão de MS. Contudo, pode-se inferir que bezerros Nelore seriam mais dependentes de alimento sólido a partir do terceiro mês de vida.

Assim, nos sistemas de produção nos quais se opte por criar animais super-precoces, que exigem maior aporte nutricional dos animais ainda lactantes, a suplementação destes deve ser iniciada a partir do $3^{\underline{0}}$ mês, como visto anteriormente, por intermédio de creep-feeding.

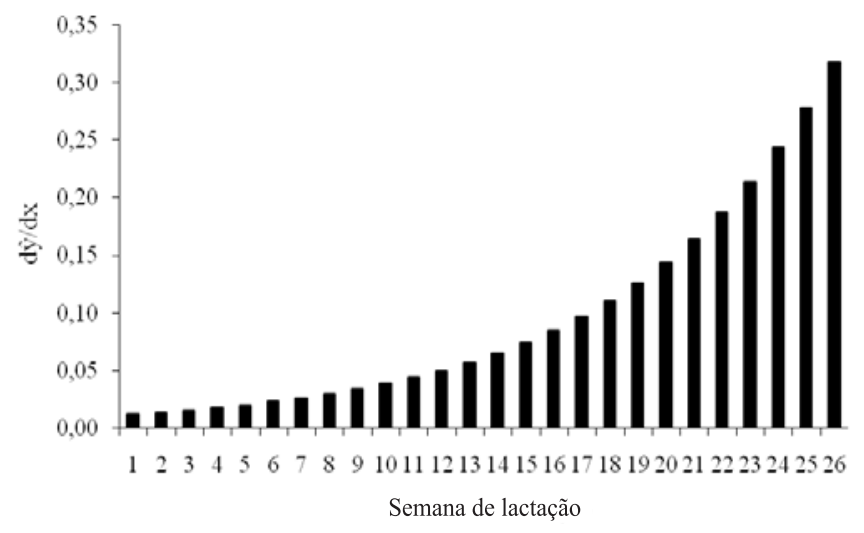

Figura 4 - Derivada da função do consumo voluntário do bezerro. 
Aparentemente, as correlações entre consumo voluntário de vaca e produção de leite ou consumo voluntário do bezerro e produção de leite são fracas, chegando a ser não significativa para a vaca. Contudo, o efeito do consumo voluntário da vaca sozinho é pouco relevante na produção de leite, bem como o efeito do consumo voluntário pelo bezerro sozinho também tem pouca influencia sobre a produção de leite. Assim, ao se analisar os efeitos conjuntamente, observa-se que à medida que se eleva o consumo voluntário da vaca obtém-se maior estimativa de produção de leite e que a produção de leite é negativamente correlacionada ao consumo voluntário do bezerro, sendo que, à medida que o consumo voluntário do bezerro aumenta, a produção de leite da vaca diminui (Tabela 4).

O desdobramento da correlação pela análise de trilha permitiu evidenciar que ambas variáveis afetaram de forma forte e direta a relação entre a produção de leite e o consumo de leite. Quanto mais leite é produzido mais se exige da vaca em consumo de nutrientes. Quanto maior a capacidade do bezerro em ingerir sólidos não-lácteos, menos se exige da vaca em termos de produção de leite. Assim, a quantidade de leite requerida por unidade de ganho de peso diminui com a idade do bezerro.

Contudo, não só a limitação do bezerro determina a forma da curva de lactação. Além disso, as características de produção de leite da vaca interferem no comportamento do bezerro, de modo que a disponibilidade de leite também pode modificar o hábito alimentar, aumentando ou diminuindo a compensação do aporte de nutrientes pelo consumo de sólidos não-lácteos.

Essa inter-relação fisiológica antagônica gera efeitos indiretos opostos aos efeitos diretos, o que culmina com correlações fenotípicas que não evidenciam a força direta dos efeitos dos consumos voluntários sobre a produção de leite.

Isto é reforçado pelo coeficiente de determinação, no qual se percebe que apenas as questões de consumo respondem por mais de $50 \%$ da variabilidade da produção de leite, considerando-se o sistema vaca $\times$ bezerro.

O ganho de peso médio diário das vacas do parto até 180 dias foi de $394 \mathrm{~g}$. No entanto, cerca de $82 \%$ desse ganho ocorreu após o pico da lactação (Figura 3). Isso possivelmente ocorre porque a lactação é fisiologicamente prioritária ao ganho de peso. Desta forma, principalmente no início da lactação, a partição de nutrientes direcionase no sentido da produção de leite.

Outro fator que parece ter grande influência sobre a produção de leite é a ordem de parto. Diversos estudos comprovam que vacas adultas apresentam produções de leite mais elevadas se comparadas a vacas primíparas. Pimentel et al. (2006) observaram efeito significativo da ordem de parto para vacas de segunda e terceira lactação relataram que resultados indicam que a maior diferença nas características da lactação de vacas primíparas e multíparas ocorre no pico da lactação.

Tabela 4 - Análise de correlação da produção de leite e consumo de matéria seca da vaca ou do bezerro

\begin{tabular}{|c|c|c|}
\hline Variável & Correlação & Valor $\mathrm{P}$ \\
\hline Consumo voluntário vaca & 0,200 & 0,169 \\
\hline Consumo voluntário bezerro & $-0,417$ & 0,019 \\
\hline \multicolumn{3}{|l|}{ Desmembramento dos fatores sobre a produção de leite } \\
\hline Efeito direto do consumo voluntário vaca & 0,831 & \\
\hline Efeito indireto de consumo voluntário vaca via consumo voluntário bezerro & $-0,631$ & \\
\hline Total - diretos e indiretos & 0,200 & \\
\hline Efeito direto do consumo voluntário bezerro & $-0,962$ & \\
\hline Efeito indireto de consumo voluntário bezerro via consumo voluntário vaca & 0,545 & \\
\hline Total - diretos e indiretos & $-0,417$ & \\
\hline Coeficiente de correlação & 0,567 & \\
\hline
\end{tabular}

\section{Conclusões}

O modelo de Jenkins \& Ferrell, com um parâmetro de ajustamento, descreve a curva de produção de leite de vacas Nelore em condições brasileiras. A produção de leite está associada ao consumo voluntário do bezerro e da vaca.

\section{Referências}

ALBUQUERQUE, L.G.; ELER, J.P.; COSTA, M.J.R.P. et al. Produção de leite e desempenho do bezerro na fase de aleitamento em três raças bovinas de corte. Revista Brasileira de Zootecnia, v.22, p.745-753, 1993.

ALENCAR, M.M.; OLIVEIRA, F.T.; TAMBASCOS, A.J. et al. Desenvolvimento pós-desmama e eficiência reprodutiva pósparto em gado de corte: influência da produção de leite. Revista Brasileira de Zootecnia, v.22, p.1012-1018, 1993. 
BRODY, S.A.; RAGSDALE, A.C.; TURNER, C.W. The rate of decline of milk secretion with the advance of the period of lactation. Journal of General Physiology, v.5, p.441-444, 1923.

CRUZ, C.D.; REGAZZI, A.J. Modelos biométricos aplicados ao melhoramento genético. 2.ed. Viçosa, MG: Gráfica Universitária - UFV, 2001. 390p.

DRAPER, N.; SMITH, H. Applied regression analysis. NewYork: John Willey \& Sons, 1966. 407p.

HALL, M.B. Calculation of non-structural carbohydrate content of feeds that contain non-protein nitrogen. Florida: University of Florida, 2000. p.A-25 (Bulletin, 339).

LANDETE-CASTILlEJOS, T.; GALLEGO, L. Technical note: The ability of mathematical models to describe the shape of lactation curves. Journal of Animal Science, v.78, p.3010-3013, 2000.

LICITRA, G.; HERNANDEZ, T.H.; Van SOEST, P.J. Standardization of procedures for nitrogen fractionation of ruminant feeds. Animal Feed Science and Technology, v.57, p.347-358, 1996.

JENKINS, T.G.; FERRELL, C.L. A note on lactation curves of crossbred cows. Animal Production, v.39, p.479-482, 1984.

JENKINS, T.G.; FERRELL, C.L. Preweaning efficiency for mature cows of breed crosses from tropically adapted Bos indicus and Bos Taurus and unadapted Bos Taurus breeds. Journal of Animal Science, v.82, p.1876-1881, 2004.

JENKINS, T.G.; FERRELL, C.L.; ROBERTS, A.J. Lactation and calf weight traits of mature cross bred cows fed varying daily levels of metabolizable energy. Journal of Animal Science, v.78, p.7-14, 2000.

PIMENTEL, M.A.; MORAES, J.C.F; JAUME, C.M. et al. Produção de leite e desempenho pós parto de vacas Hereford em distintas condições reprodutivas criadas extensivamente. Ciência Rural, v.35, p.150-156, 2005.

MASSELIN, S.; SAUVANT, D.; CHAPOUTOT, P. et al. Les modèles d'ajustement des courbes de lactation. Annales de Zootechnie, v.36, p.171-206, 1987

MERTENS, D.R. Gravimetric determination of amylase-treated neutral detergent fiber in feeds with refluxing in beaker or crucibles: collaborative study. Journal of AOAC International, v.85, p.1217-1240, 2002.
McCARTER, M.N.; BUCHANAN, D.S.; FRAHM, R.R. Comparison of crossbred cows containing various proportions of Brahman in spring or fall calving systems: II. Milk production. Journal of Animal Science, v.69, p.77-84, 1991.

NATIONAL RESEARCH COUNCIL - NRC. Nutrient requirements of beef cattle. 7.ed. Washington, D.C.: National Academic Press, 1996. 242p.

NELDER, J.A. Inverse polynomials, a useful group of multi-factor response functions. Biometrics, v.22, p.128-141, 1966.

RESTLE, J.; PACHECO, P.S.; PADUA, J.T. et al. Efeito da taxa de ganho de peso pré-desmama de bezerras de corte e do nível nutricional pós-parto, quando vacas, sobre a produção e composição do leite e o desempenho de bezerros. Revista Brasileira de Zootecnia, v.34, p.197-208, 2005.

SHERCHAND, L.; McNEW, R.W.; KELLOGG, D.W. et al. Selection of a mathematical model to generate lactation curves using daily milk yields of Holstein cows. Journal of Dairy Science, v.78, p.2507-2513, 1995.

SILVA, D.J.; QUEIROZ, A.C. Análise de alimentos (métodos químicos e biológicos). 3.ed. Viçosa, MG: Imprensa Universitária - UFV, 2002. 165p.

SIKKA, L.C. A study of lactation as affected by heredity and environment. Journal of Dairy Research, n.17, p.231-252, 1950.

SOUZA, G.S. Introdução aos modelos de regressão linear e não-linear. Brasília: Embrapa-SPI, 1998. 505p.

STATISTICAL ANALYSES SYSTEM - SAS. SAS user's guide. Cary: SAS, 1999. v.8, 295p.

VAN SOEST, P.J.; ROBERTSON, J.B. Systems of analysis for evaluating fibrous feeds. In: PIGDEN, W.J.; BALCH, C.C.; GRAHAM, M. (Eds.) Standardization of analytical methodology for feeds. Ottawa: International Development Research Centre, 1980. p.49.

VAN SOEST, P.J.; ROBERTSON, J.B.; LEWIS, B.A. Methods for dietary fiber, neutral detergent fiber, and nonstarch polysaccharides in relation to animal nutrition. Journal of Animal Science, v.74, p.3583-3597, 1991.

WOOD, P.D.P. Algebraic model of the lactation curve in cattle. Nature, n.216, p.164-165, 1967. 